\title{
The Cochrane Collaboration: Cochrane Airways Group
}

\author{
F S F Ram, S J Milan, P W Jones
}

I $\mathrm{t}$ is well known that health care professionals, consumers, researchers and policy makers are over-

whelmed with unmanageable amounts of information. In an influential book ${ }^{1}$ published in 1972, Archie Cochrane, a British epidemiologist, drew attention to our great collective ignorance about the effects of health care. He recognised that people who want to make more informed decisions about health care do not have ready access to reliable reviews of the available evidence. In 1979, he wrote: ${ }^{2}$

"It is surely a great criticism of our profession that we have not organised a critical summary, by speciality or subspecialty, adapted periodically, of all relevant randomised controlled trials".

In 1987, the year before Cochrane died, he referred to a systematic review ${ }^{3}$ of randomised controlled trials (RCTs) of care during pregnancy and childbirth as "a real milestone in the history of randomised trials and in the evaluation of care", and suggested that other specialities should copy the methods used. In the same year, the scientific quality of many published reviews was shown to leave much to be desired. ${ }^{4}$ As Cochrane had emphasised, reviews of research evidence must be prepared systematically and they must be kept up-todate to take account of new evidence. If this is not done, important effects of health care (good and bad) will not be identified promptly, and consequently people using the health services will be ill served. In addition, without systematic, up-to-date reviews of previous research, plans for new research will not be well informed. Therefore, researchers and funding bodies will miss promising leads, and embark on studies asking questions that have already been answered ${ }^{5}$. The Cochrane Collaboration has developed in response to Cochrane's call for systematic, up-to-date reviews of all relevant RCTs of health care. Cochrane's suggestion that the methods used to prepare and maintain reviews of controlled trials in pregnancy and childbirth should be applied more widely was taken up by the Research and Development Programme, initiated to support the United Kingdom's National Health Service. Funds were provided to establish a 'Cochrane Centre', to collaborate with others, in the UK and elsewhere, to facilitate systematic reviews of randomised controlled trials across all areas of health care. ${ }^{6,7}$ In October 1993 - at what was to become the first in a series of annual Cochrane Colloquia - 77 people from eleven countries co-founded 'The Cochrane Collaboration'. The Cochrane Collaboration is an international organisation that aims to help people make well-informed decisions about healthcare by preparing, maintaining and promoting the accessibility of systematic reviews of the effects of healthcare interventions. It is a not-for-profit organisation, established as a company, limited by guarantee, and registered as a charity in the UK.

The Cochrane Collaboration is now eight years old, and in that short period over one thousand systematic reviews have been published on the Cochrane Library. It is estimated that the current total may double over the next two years.

\section{Cochrane Airways Group}

The Airways Group of the Cochrane Collaboration was established in 1995, when eleven other review groups were already in existence. By the end of 2000 the number had increased to nearly 50 , covering virtually all areas of healthcare. The Cochrane

Collaboration has grown into a highly successful, world-wide initiative and the Airways Group has in excess of 300 active members in 21 countires. Our membership represents the fields of medicine, nursing, health psychology, physiotherapy, psychotherapy and statistics. The introduction of the group's website (http://www.cochrane-airways.ac.uk) in 1999 provided a significant development in our support to group members.

The Airways Group has an editorial base (at St. George's Hospital Medical School), whose role is to support the development and maintenance of Airways Group reviews. The editorial base staff provide comprehensive methodological and software support, identify all trials through electronic searches, obtain papers from medical libraries, obtain translations of all relevant non-English trials, process all reviews and protocols for publication and provide the planning and coordinating function for this large international group. We now have 80 reviews and 81 protocols published on the Cochrane Library. We expect to have completed over 100 reviews by the end of 2002 .

Archie Cochrane made the point that reviews must be kept up-to-date to take account of new evidence. The Airways Group has now entered a phase of both continued growth (with greater than 20 new reviews per year) and consolidation (updating existing reviews). Updating a review is not always as demanding as developing the original, although that is not always the case, since the introduction of a new type of therapy often shows an almost exponential growth in trial publication over the following years.

Over the last four years we have actively promoted the dissemination of our completed systematic reviews, beyond publication on the Cochrane Library. A number of Airways Group reviews have been published secondarily in other peer-reviewed journals and they

\section{Felix S F Ram}

Respiratory Research

Fellow, Netherlands

Astma Fonds

\section{Stephen J Milan}

Review Group

Co-ordinator, Cochrane

Airways Group

\section{Paul W Jones}

Professor of Respiratory Medicine \& Co-ordinating Editor, Cochrane Airways Group

\section{St. Georges Hospital}

Medical School,

Department of

Physiological Medicine,

London, UK

Correspondence to:

Felix S F Ram

Respiratory Research Fellow

St. Georges Hospital

Medical School

Department of

Physiological Medicine

Level 0, Jenner Wing

Cranmer Terrace, Tooting

London, SW17 0RE

fram@sghms.ac.uk

Prim Care Respir J 2001: 10(2);45-46 
have also received oral or poster presentations at major respiratory conferences in Europe, USA and Australia. We held our first international symposium on evidence-based respiratory medicine in 1999 at the Royal Society of Medicine. We are currently considering the feasibility of repeating the event every four years with the next one planned for the autumn of 2003.

The Airways Group reviews are providing much important material for the BTS/SIGN evidence based asthma and COPD guidelines, and the reviews are having a similar impact in Australia and Canada. A recent paper ${ }^{8}$ highlights the quality of Airways Group's systematic reviews, and the growing international reputation of the group's work which reflects the rigour that goes into the development of such work.

The Collaboration is built on ten principles: collaboration, enthusiasm, avoiding duplication, minimizing bias, keeping up-to-date, ensuring relevance, ensuring access, continually improving the quality of its work, continuity and enabling wide participation. Its to help people make well informed decisions about health care by preparing, maintaining and ensuring the accessibili- ty of systematic reviews of the effects of health care interventions. To summarise, the Cochrane

Collaboration has evolved rapidly since it was inaugurated at the 1st Colloquium, but its basic objectives

and principles have remained the same as they were at its inception.

\section{REFERENCES}

1. Cochrane AL. Effectiveness and Efficiency.Random reflections on health services. London: Nuffield Provincial Hospitals Trust, 1972. (Reprinted in 1989 in association with the BMJ).

2. Cochrane AL. 1931-1971: a critical review, with particular reference to the medical profession. In: Medicines for the year 2000 London: Office of Health Economics, 1979,1-11

3. Cochrane AL. Foreword. In: Chalmers I, Enkin M, Keirse MJNC, eds. Effective care in pregnancy and childbirth. Oxford: Oxford University Press, 1989.

4. Mulrow CD. The medical review article: state of the science. Ann Int Med 1987;106:485-8.

5. Antman EM, Lau J, Kupelnick B, Mosteller F, Chalmers TC. A comparison of results of meta-analyses of randomized control trials and recommendations of clinical experts. JAMA 1992;268: 240-8.

6. Chalmers I, Dickersin K, Chalmers TC. Getting to grips with Archie Cochrane's agenda. BMJ 1992;305:786-8.

7. Lancet Editorial. Cochrane's Legacy. Lancet 1992;340:1131-2.

8. Jadad AR, Moher M, Browman GP, Booker L, Sigouin C, Fuentes M, Stevens R. Systematic reviews and meta-analyses on the treatment of asthma: a critical evaluation. BMJ 2000;320: $537-40$

\section{SCOOP FIRST PRIZE IN ASTHMA COMPETITION}

Are you helping people with asthma actively take control of their condition? Have you developed a programme that encourages good communication and multi- agency working across primary and secondary care? If so, tell us about it.

The Asthma Journal is pleased to invite entries for the 2001 Henry Blair Prize.

The competition is open to health professionals (individuals or groups) working in the UK who have helped to develop initiatives that improve asthma care in their area, initiatives which will really make a difference.

The prize, to the value of $£ 600$, will be awarded for the local initiative that is judged to target asthma care most effectively, particularly in the areas specified below:

a) Initiatives which encourage patient involvement in their own care eg self management programmes

b) Projects developing the interface between primary and secondary care eg care pathways

However, consideration will also be given to other innovative programmes.

Entrants are asked to describe in up to 1200 words the key points of improving asthma care in their area.

For further details and an application form, please contact: Kate Jewitt, Professional Relations Officer, National Asthma Campaign, Providence House, Providence Place, London N1 ONT. Tel: +44 (0)20 77045830 Fax: +44 (0)20 77040740 Email: kjewitt@asthma.org.uk

Closing date for entries: September 10th 2001

\section{Dr Henry Blair}

This prize has been funded by friends and colleagues of the late Dr Henry Blair, with the aim of stimulating improvements in the care of patients with asthma. Dr Blair spent a long professional career in East London, working as a general practitioner and hospital allergist. In both of these roles he maintained an active clinical and research interest in asthma, and Dr Blair's published work provides some of the best follow-up data that we have on outcomes in children with asthma. Even after his retirement Dr Blair maintained his interest in asthma. Shortly before his death in 1984 he officially opened, as Guest of Honour, the Waltham Forest Branch of what was then the Asthma Society, now the National Asthma Campaign. 